\title{
The Effect of Tissue Flossing Technique on Sports and Injury Prevention and Rehabilitation: A Systematic Review of Recent Research
}

\author{
Gao Jianhong ${ }^{1}$, Chee Chen Soon ${ }^{1, *}$, Thung Jin Seng ${ }^{2}$, Zeinab Zaremohzzabieh ${ }^{1}$, \\ Shamsulariffin Samsudin ${ }^{1}$ \\ ${ }^{1}$ Faculty of Educational Studies, University Putra Malaysia, Serdang, 43400, Selangor, Malaysia \\ ${ }^{2}$ National Sports Institute of Malaysia, Kompleks Sukan Negara, Bukit Jalil, Kuala Lumpur, Malaysia
}

Received July 28, 2021; Revised September 6, 2021; Accepted October 6, 2021

\section{Cite This Paper in the following Citation Styles}

(a): [1] Gao Jianhong, Chee Chen Soon, Thung Jin Seng, Zeinab Zaremohzzabieh, Shamsulariffin Samsudin, "The Effect of Tissue Flossing Technique on Sports and Injury Prevention and Rehabilitation: A Systematic Review of Recent Research," International Journal of Human Movement and Sports Sciences, Vol. 9, No. 6, pp. 1157 - 1173, 2021. DOI: 10.13189/saj.2021.090611.

(b): Gao Jianhong, Chee Chen Soon, Thung Jin Seng, Zeinab Zaremohzzabieh, Shamsulariffin Samsudin (2021). The Effect of Tissue Flossing Technique on Sports and Injury Prevention and Rehabilitation: A Systematic Review of Recent Research. International Journal of Human Movement and Sports Sciences, 9(6), 1157 - 1173. DOI: 10.13189/saj.2021.090611.

Copyright $\bigcirc 2021$ by authors, all rights reserved. Authors agree that this article remains permanently open access under the terms of the Creative Commons Attribution License 4.0 International License

\begin{abstract}
Flossband, as a novel and effective tissue flossing technique, is becoming increasingly popular in the field of athletic training, sports injury prevention, and rehabilitation. The purpose of this literature review is to summarize updated evidence about the effects of flossband application on joint range of motion (ROM), pain, muscle tightness, strength, and physical functional performance as well as identify research gaps for future study. Google Scholar, PubMed, EBSCO, and Web of Science were used to search related articles. The keywords of floss bands, flossbands, floss band, tissue flossing, flossing band, voodoo floss band, voodoo floss bands, track floss, rock floss, life floss band, and Rogue voodoo floss were used to extract target articles. English journal articles, full-text available, and content related to outcome measures were included. Conference abstracts, books, case studies, guideline reviews were omitted. 23 full-text journal articles were included for further qualitative analysis after removing duplicates and deleting articles that violate the screening criterion. Flossband application on limbs, soft tissue, or joints with about $50 \%$ flossing tension or 150 $\mathrm{mmHg}$ wrapping pressure could have small to medium effects on the parameters of ROM, muscle stiffness, muscle strength, and physical function performance, and large effects on pain management. Most previous studies were
\end{abstract}

mainly focused on the acute effect of flossband application on peripheral joints or soft tissues in healthy and active participants or well-trained athletes. Therefore, for future studies, more evidence is needed on the benefits of long-term flossband trunk application and concerning patients with various diseases.

Keywords Flossband, Pain Management, Range of Motion, Tightness, Strength, Physical Function Performance, Fascial Release

\section{Introduction}

High-energy sports are prone to sports injuries as a result of tissues being overused during intense sports activities. Repeated exposure to the high mechanical stresses associated with sporting activities promotes abnormal postural adjustments and myofascial injuries [1]. Inflammatory reactions to tissue injury can change the structure of myofascial tissues, thus causing discomfort and hypersensitivity, and reducing an athlete's range of motion (ROM), strength and performance [2]. Many therapeutic strategies have been used to prevent and cure 
sports injuries and dysfunctions caused by biomechanical deficiencies and overuse. A flossband treatment is among the most popular therapeutic interventions in sports [3]. The anecdotal usage of this treatment among athletic individuals is becoming a popular technique to increase joint ROM, enhance both injury prevention and recovery, and improve overall sports performance. The soft tissue flossing technique is a treatment tool and an optional strategy to assist in sports training [4]. A flossband is composed of multiple layers of natural rubber, with a thickness of more than 1 centimeter, and this technique is an easy-to-acquire skill by different occupational professionals [5]. In addition, flossband is cheap, convenient, flexible, and effective [6].

Over the past few years, flossband application has been more commonly used in the field of training or rehabilitation. Accordingly, researchers have concentrated on testing the effects of flossband application on joint ROM in different joints [7,8], muscle tightness [9,10], muscle strength $[15,16]$, as well as physical function performance [8,11-13]. However, despite the apparent benefits found in the above parameters, there was no relevant literature review that integrated these different findings. Wrapping tension or compression pressure of flossband application was the key skill. By reviewing the previous studies, these studies investigated the effects of flossband application by using various flossing tensions or wrapping pressures, such as 50\% stretch tension [5,14] and $150-200 \mathrm{mmHg}$ wrapping pressure $[15,16]$ on the different soft tissues or joints. However, they all observed similar outcome measures. Unfortunately, there is little evidence overall for this technique and the current evidence. Very few studies showed either positive or negative support for use of tissue flossing.

To date, several papers have been published about the effects of tissue flossing techniques on the functionality of the hamstring muscles [9], ankle ROM and tensiomyography parameters [16], or the healthy and impaired musculoskeletal system [17]. No such overview exists for the effects of flossing. Thus, an in-depth review on the effects of the tissue flossing method in sports injury prevention and rehabilitation is required. Going forward, the purpose of this literature review was to collect, analyze and summarize the recent research on the application progress of tissue flossing technique, as well as to provide updated evidence about the effects of applying flossing technique on the parameters of pain, joint ROM, muscle tightness and physical functional performance in sports and clinical practice. Moreover, this literature review also aimed to identify potential research areas for future studies on the tissue flossing technique.

\section{Methodology}

This review included research studies related to the outcomes of tissue flossing technique application on different peripheral joints and soft tissues in different groups of people, such as general healthy young people, athletes, or patients. Hence, our population consisted of studies across seven years from January 2014 to May 2021.

As part of the search strategy, Google Scholar, PubMed, EBSCO, and Web of Science were used to search the related articles. The keywords of floss bands, flossbands, floss band, tissue flossing, flossing band, voodoo floss band, voodoo floss bands, track floss, rock floss, life floss band, and Rogue voodoo floss were used separately or in combination to extract the target articles (Table 1). The articles were required to be journal articles that were published in English. Conference abstracts, related books, case studies, guideline reviews, unavailable full text or articles without any relationship to the content by reviewing titles and abstracts were excluded. In addition, articles which did not report the outcome measures of pain, joint range of motion, and functional performance were also omitted.

Table 1. Search Strategy.

\begin{tabular}{|c|l|c|}
\hline Type of Database & \multicolumn{1}{|c|}{ Searching type } & Result \\
\hline PubMed & $\begin{array}{l}\text { (floss bands) OR (flossbands)) OR (floss band)) OR (tissue flossing)) OR (flossing band)) OR (voodoo } \\
\text { floss band)) OR (voodoo floss bands)) OR (track floss)) OR (rock floss)) OR (life floss band)) OR (Rogue } \\
\text { voodoo floss) }\end{array}$ & 195 \\
\hline EBSCO & $\begin{array}{l}\text { AB floss bands OR AB flossbands OR AB floss band OR AB flossing band OR AB tissue flossing OR AB } \\
\text { voodoo floss band OR AB voodoo floss bands OR AB track floss OR AB rock floss OR AB life floss band } \\
\text { OR Rogue voodoo floss }\end{array}$ & 14 \\
\hline Wed of Science & $\begin{array}{l}\text { TI = (floss bands OR flossbands OR floss band OR flossing band OR tissue flossing OR voodoo floss } \\
\text { band OR voodoo floss bands OR track floss OR rock floss OR life floss band OR Rogue voodoo floss) }\end{array}$ & 19 \\
\hline Google scholar & $\begin{array}{l}\text { "floss bands" OR "flossbands" OR "floss band" OR "flossing band" OR "tissue flossing" OR "voodoo } \\
\text { floss band" OR "voodoo floss bands" OR "track floss" OR "rock floss" OR "life floss band" OR "Rogue } \\
\text { voodoo floss" }\end{array}$ & 182 \\
\hline
\end{tabular}




\subsection{Study Selection}

Two reviewers worked on the task of article searching and article quality evaluation. On one aspect, they individually used the same keywords to search articles in these four search engine databases. Then, they reviewed the title, abstract and full-text articles according to the inclusion and exclusion criteria. Articles were included in this study when the content is about the effects of tissue flossing on different soft tissues and various peripheral joints such as hamstring, upper limb muscles, quadriceps, Achilles tendon, ankle joint, knee joint, shoulder joint, elbow joint, and so on. Additionally, the articles should be focused on the parameters of pain, joint range of motion, muscle tightness, muscle strength, and physical function performance. For the other aspect, the two reviewers independently used methodology checklists which were developed by the Scottish Intercollegiate Guidelines Network (SIGN) (https://www.sign.ac.uk/checklists-and-notes.html) to evaluate the quality of the articles [18].

\section{Results}

This research identified 415 related articles in total. Of these, 410 articles were obtained from databases of PubMed, EBSCO, Web of Science, and Google Scholar by using the related keywords and search strategies. In addition, 1 abstract and 4 journal articles were found from other resources. Next, 85 articles were removed due to duplication. After that, 307 articles were excluded because these articles were not in English, had unrelated content, were not journal articles (news, product introduction, advertising, or conference abstracts) or the full-text was unavailable. Finally, 23 full-text journal articles were included for further qualitative analysis by following the exclusion criterion and inclusion criterion through a rigorous screening process (see Figure 1). Table 2 summarizes the results of accessible studies.
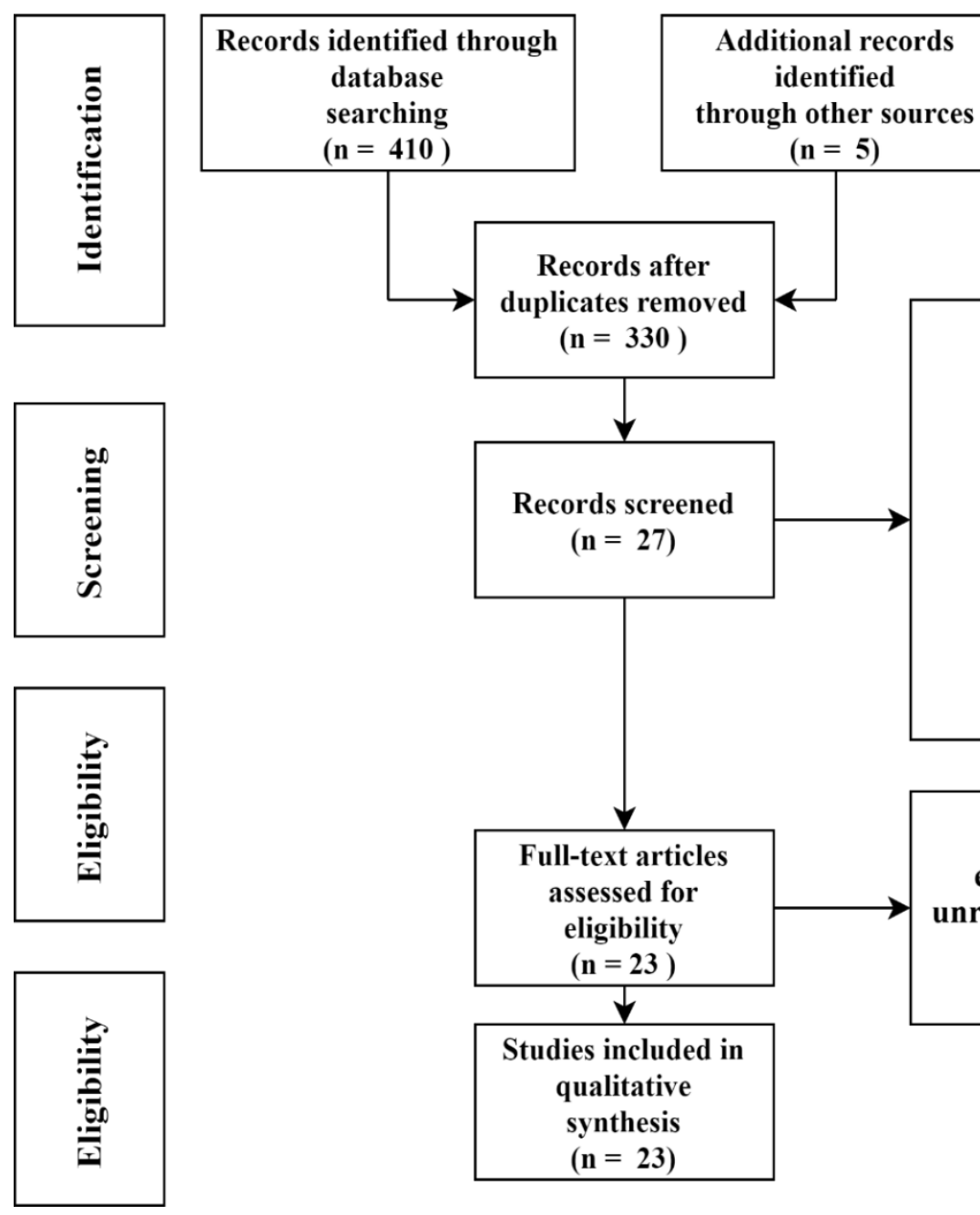

Records after duplicates removed $(\mathbf{n}=330)$ $(\mathbf{n}=\mathbf{5})$

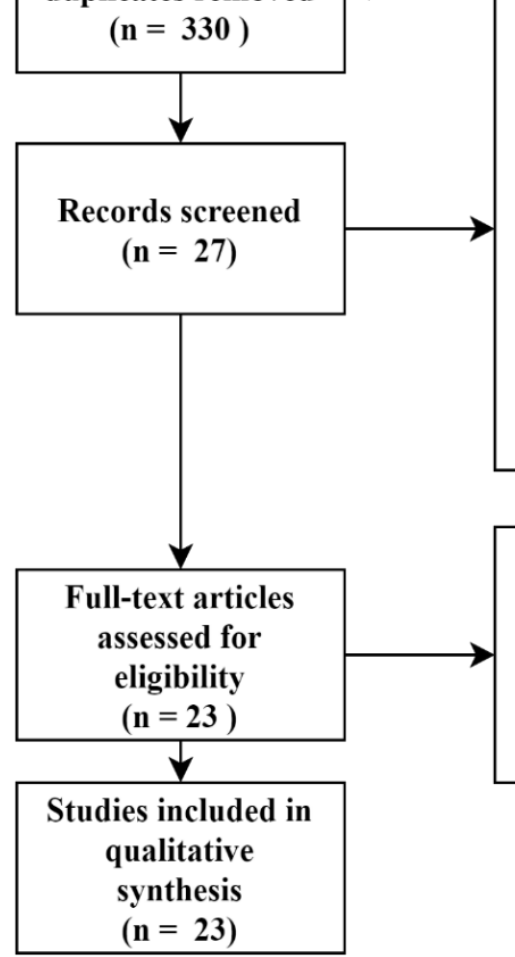

Records excluded $(n=303)$

Non-English $(n=32)$

Title/abstract $(\mathrm{n}=\mathbf{2 4 5})$

Abstract /guideline ( $\mathbf{n}=\mathbf{2 0})$

Case study $(n=4)$

Full text unavailable $(\mathbf{n}=\mathbf{2})$

Books ( $\mathrm{n}=1)$

Thesis or Dissertation $(n=6)$

Full-text articles

excluded, with reasons unrelated outcome measures

$(n=4)$

Figure 1. Searching result 
Table 2. The result summary of previous studies

\begin{tabular}{|c|c|c|c|c|c|c|c|}
\hline $\begin{array}{c}\text { Author and } \\
\text { year }\end{array}$ & Study design & $\begin{array}{c}\text { Sample size/ } \\
\text { group }\end{array}$ & $\begin{array}{c}\text { Application } \\
\text { part }\end{array}$ & Band and application & Test and Intervention & $\begin{array}{l}\text { Outcome } \\
\text { Measure }\end{array}$ & Conclusion \\
\hline Driller [19] & Experimental & $\begin{array}{l}69 \text { healthy } \\
\text { recreational } \\
\text { athletes } \\
\text { ( } 32 \text { males and } 37 \\
\text { females) } \\
\text { Random } \\
\text { split into two } \\
\text { groups; } \\
\text { Floss: } 38 \\
\text { Control: } 31\end{array}$ & Ankle & $\begin{array}{l}\text { Life Flossband } \\
\text { Floss group: wrapped floss } \\
\text { band on both ankles (figure } \\
8 \text { wrapping method on } \\
\text { ankle) } \\
\text { Control group: without } \\
\text { floss band } \\
\text { Compression force } 178 \pm 18 \\
\text { mmHg }\end{array}$ & $\begin{array}{l}\text { Pre-Posttest (post-testing were tested after 15, } \\
30 \text {, and } 45 \text { minutes) } \\
\text { Both groups were given standard } 5 \text { minutes' } \\
\text { warm-up and bodyweight low limb dynamic } \\
\text { stretch. Then floss group was applied flossband } \\
\text { in both ankle and was asked to do } 2 \text { minutes' } \\
\text { ankle full AROM of dorsiflexion and Plantar } \\
\text { flexion. Control group was done the same } \\
\text { exercise without flossband. After } 2 \text { minutes } \\
\text { ankle exercise, two group were instructed to } \\
\text { stand up and walk around for one minute and did } \\
\text { the post-test }\end{array}$ & $\begin{array}{l}\text { WBLT, CMJ, } \\
\text { 5-m Sprint (secs) } \\
\text { 10-m Sprint } \\
\text { (secs) } \\
\text { 15-m Sprint } \\
\text { (secs). }\end{array}$ & $\begin{array}{l}\text { No significance difference } \\
\text { between floss group and control } \\
\text { group during WBLT, CMJ and } \\
\text { sprint, but there was a trend floss } \\
\text { band benefit to ROM, CMJ and } \\
\text { sprint performance. Significant } \\
\text { improvement to WBLT after the } \\
\text { application of CTF for up to } \\
45 \text { min. }\end{array}$ \\
\hline $\begin{array}{l}\text { Driller and } \\
\text { Overmayer } \\
{[15]}\end{array}$ & Experimental & $\begin{array}{l}52 \text { healthy } \\
\text { recreational } \\
\text { athletes }((29 \\
\text { males and } 23 \\
\text { females) } \\
\text { Randomly assign; } \\
\text { Floss group: } 26 \\
\text { Control group: } 26\end{array}$ & Ankle & $\begin{array}{l}\text { Life Flossband } \\
\text { Floss group: wrapped floss } \\
\text { band on left/right side } \\
\text { figure } 8 \text { wrapping method } \\
\text { on ankle) } \\
\text { Control group: without } \\
\text { floss band } \\
\text { Compression force } 178 \pm 18 \\
\mathrm{mmHg}\end{array}$ & $\begin{array}{l}\text { Pre-Posttest (acute effect) } \\
\text { Floss group: flossband was applied on one side } \\
\text { of the ankle, and participants were asked to do } \\
\text { both sides ankle full AROM of dorsiflexion and } \\
\text { Plantarflexion, } 20 \text { repetitions within } 2 \text { minutes } \\
\text { Control group: did the same exercise without } \\
\text { applying flossband }\end{array}$ & $\begin{array}{l}\text { WBLT, } \\
\text { ROM(Dorsiflexi } \\
\text { on/plantarflexion } \\
\text { ), } \\
\text { Single-leg jump } \\
\text { height and } \\
\text { velocity }\end{array}$ & $\begin{array}{l}\text { No significant difference } \\
\text { between the floss group and } \\
\text { control group during WBLT, } \\
\text { ROM, jump height and jump } \\
\text { velocity, but it was a significant } \\
\text { difference between pre and } \\
\text { post-test in floss group. }\end{array}$ \\
\hline Hagen [11] & Experimental & $\begin{array}{l}32 \text { healthy college } \\
\text { student } \\
\text { (21 males and } 15 \\
\text { females) } \\
\text { Age: } 22.1 \pm 2.4 \\
\text { Randomly assign } \\
\text { into } 4 \text { groups: } \\
\text { Control (without } \\
\text { treatment) } 8 \\
\text { DYN (CKC } \\
\text { dynamic } \\
\text { stretching) } 8 \\
\text { STAT (CKC } \\
\text { statistic } \\
\text { stretching) } 8 \\
\text { Floss (CKC } \\
\text { dynamic } \\
\text { combined with } \\
\text { wrapping } \\
\text { flossband) } 8 \\
\end{array}$ & Ankle & $\begin{array}{l}\text { Rogue VooDoo Flossband } \\
\text { (Columbus, OH) } \\
\text { Floss group (figure } 8 \\
\text { wrapping method on ankle) }\end{array}$ & $\begin{array}{l}\text { Pre-Posttest (post-testing were testing } \\
\text { immediate effect, } 4,8 \text {, and } 12 \text { minutes) } \\
\text { Control: sat and rest } 10 \text { minutes } \\
\text { DYN ( } 20 \text { reps/side, both sides, hold } 1 \mathrm{~s}-2 \mathrm{~s}, 2 \text { sets) } \\
\text { STAT (holds } 45 \text { seconds/side, both sides, } 2 \text { sets) } \\
\text { Floss group (wrapped flossband on both ankles, } \\
20 \text { reps/side, both sides, hold } 1 \mathrm{~s}-2 \mathrm{~s}, 2 \text { sets) }\end{array}$ & $\begin{array}{l}\text { DF ROM } \\
\text { (Lunge test, } \\
\text { seated open } \\
\text { chain) } \\
\text { Bodyweight } \\
\text { overhead squat } \\
\text { test }\end{array}$ & $\begin{array}{l}\text { No significant differences } \\
\text { among groups during these } \\
\text { outcome measures. Significant } \\
\text { improvement in floss group } \\
\text { during ROM OF CKC DF and } \\
\text { Bodyweight overhead squat test. } \\
\text { Significant improvement in } \\
\text { DYN during DF ROM OF CKC } \\
\text { and OKC. }\end{array}$ \\
\hline
\end{tabular}


Table 2. Continued

\begin{tabular}{|c|c|c|c|c|c|c|c|}
\hline $\begin{array}{l}\text { Kiefer et al. } \\
{[20]}\end{array}$ & Pilot & $\begin{array}{l}60 \text { Healthy } \\
\text { college students } \\
\text { (18-24 years old) } \\
\text { Randomly } \\
\text { assigned into } 2 \\
\text { groups } \\
\text { Control group 30, } \\
\text { Floss group 30 }\end{array}$ & Upper Arm & $\begin{array}{l}\text { Voodoo flossband } \\
\text { Floss group (50\% tension } \\
\text { applied vertical to the } \\
\text { deltoid and directly contact } \\
\text { with skin on the dominant } \\
\text { arm) } \\
\text { Control group (applied } \\
\text { underwrap directly to skin) }\end{array}$ & $\begin{array}{l}\text { Pre-posttest } \\
\text { Floss group (seated position, should stretch with } \\
\text { wearing floss band, } 30 \text { secs/set, } 5 \text { set) } \\
\text { Control group (seated position, should stretch } \\
\text { with underwrap, } 30 \text { secs/set, } 5 \text { set) }\end{array}$ & $\begin{array}{l}\text { GH flexion ROM } \\
\text { in a seated } \\
\text { position } \\
\text { Participants' } \\
\text { perception score } \\
\text { of flexibility }\end{array}$ & $\begin{array}{l}\text { No significant between groups } \\
\text { during outcomes measures, but } \\
\text { there was a significant between } \\
\text { pre and post-test in floss group. }\end{array}$ \\
\hline $\begin{array}{l}\text { Ross and } \\
\text { Kandassam } \\
{[21]}\end{array}$ & Cross-over & $\begin{array}{l}10 \text { healthy } \\
\text { participants (male } \\
5 \text {, female 5) Age: } \\
23.8 \pm 4.66\end{array}$ & $\begin{array}{l}\text { Achilles } \\
\text { Tendon }\end{array}$ & $\begin{array}{l}\text { Voodoo flossband } \\
(50 \% \text { tension, from } 2 \mathrm{~cm} \\
\text { superior to the calcaneal } \\
\text { towards much belly of } \\
\text { gastrominus) } \\
\text { Flossing two legs in a } \\
\text { different session. }\end{array}$ & $\begin{array}{l}\text { Pre-posttest (posttest included: immediately } \\
\text { effect and after intervention } 7 \text { hours) } \\
\text { There were two separate sessions. } \\
\text { Randomly choose one side of ankle wrapping } \\
\text { flossband for } 150 \text { secs and did full ROM DF } \\
\text { exercises, such as deep squat and calf raise. } \\
\text { Then after } 72 \text { hours of washout, another ankle } \\
\text { applied the same method }\end{array}$ & DF ROM & $\begin{array}{l}\text { A trend of immediate effect } \\
\text { flossband intervention can be a } \\
\text { benefit to DF ROM, }\end{array}$ \\
\hline $\begin{array}{l}\text { Stevenson } \\
{[22]}\end{array}$ & Cross-over & $\begin{array}{l}5 \text { recreational } \\
\text { male athletes } \\
\text { Who had } \\
\text { Tightness, } \\
\text { stiffness, heel } \\
\text { pain, Achilles } \\
\text { tendon pain or } \\
\text { calf pain (Age: } \\
\text { 18) } \\
\text { Random assign } \\
\text { into floss group } \\
\text { and control group }\end{array}$ & Ankle & $\begin{array}{l}\text { Flossband (180 mmHg, } \\
\text { wrapped start at midfoot } \\
\text { until inferior soleus) }\end{array}$ & $\begin{array}{l}\text { Pre-posttest } \\
\text { Both leg did } 2 \text { minutes' exercise (included ankle } \\
\text { DF/PF/circle, calf raise, body weight squat) } \\
\text { Floss group wrapped flossband to do the } \\
\text { exercise, control group without any wrapping }\end{array}$ & $\begin{array}{l}\text { Ankle ROM (DF, } \\
\text { PF), WBLT with } \\
\text { knee SL and BL, } \\
\text { TIGHT }\end{array}$ & $\begin{array}{l}\text { There was significant difference } \\
\text { between floss group and control } \\
\text { groups during DF ROM, and } \\
\text { there was a trend flossband that } \\
\text { may help WBLT comparing with } \\
\text { the control group. }\end{array}$ \\
\hline $\begin{array}{l}\text { Williams } \\
\text { [23] }\end{array}$ & Experimental & $\begin{array}{l}16 \text { healthy } \\
\text { participants } \\
\text { (Male: } 4 \text {, } \\
\text { Female:12), } \\
\text { randomly } \\
\text { assigned into: } \\
\text { Control:5 people } \\
\text { IASTM: } 6 \\
\text { Floss group } 5\end{array}$ & Ankle & $\begin{array}{l}\text { Floss group: applied } \\
\text { standard ankle tape method } \\
\text { ( } 8 \text { figure) } \\
\text { Wrapped non-dominant } \\
\text { ankle }\end{array}$ & $\begin{array}{l}\text { Pre-posttest (posttest was tested after } \\
\text { intervention } 1 \mathrm{~W}, 2 \mathrm{~W}, 3 \mathrm{~W} \text { and } 4 \mathrm{~W} \text { ) } \\
\text { Control group: calf stretch } 30 \mathrm{secs} / \mathrm{set}, 3 \text { sets } \\
\text { (Slant board), calf raise exercise 10reps/set, } \\
\text { 3sets, } 2 / \text { week, } 4 \text { week } \\
\text { IASTM applied HawkGrips IASTM tools for } 5 \\
\text { minutes, and then did the same exercise like } \\
\text { control group, } 2 / \text { week, } 4 \text { week } \\
\text { Floss group: } 2 \text { sets of } 20 \mathrm{DF} \text { and PF with } \\
\text { applying flossband, and then did the same } \\
\text { exercise that the control group did. }\end{array}$ & Ankle DF ROM & $\begin{array}{l}\text { There was no significant } \\
\text { difference between groups } \\
\text { during ankle DF ROM over time, } \\
\text { but there was a significant } \\
\text { improvement over time in each } \\
\text { group. }\end{array}$ \\
\hline
\end{tabular}


Table 2. Continued

\begin{tabular}{|c|c|c|c|c|c|c|c|}
\hline $\begin{array}{l}\text { Cheatham } \\
\text { [5] }\end{array}$ & Experimental & $\begin{array}{l}30 \text { participants } \\
\text { (male } 15, \\
\text { female15) } \\
\text { randomly } \\
\text { assigned into: } \\
\text { Foam roller } \\
\text { group: } 10 \\
\text { Soft tissue } \\
\text { mobilization } \\
\text { group:10 } \\
\text { Floss group: } 10\end{array}$ & Thigh & $\begin{array}{l}\text { Rockfloss }{ }^{\circledR} \text { Flossband } \\
50 \% \text { tension } \\
\text { Applied from upper patella } \\
\text { to AIIS. }\end{array}$ & $\begin{array}{l}\text { Pre-posttest } \\
\text { Foam roller group: left side quadriceps release } \\
\text { by using foam roller } 2 \text { minutes } \\
\text { Soft tissue mobilization group: tool of soft tissue } \\
\text { mobilization on left side quadriceps, inclined } \\
45^{\circ} \text { on the skin, } 2 \text { minutes } \\
\text { Floss group: flossband wrap covered the } \\
\text { quadriceps muscles above the patella to below } \\
\text { AIIS combined exercises (hip flexion } 30 \text { secs, } \\
\text { knee flexion and extension } 30 \text { secs, bodyweight } \\
\text { squat } 1 \text { minutes), (50\% tension), } 2 \text { minutes }\end{array}$ & $\begin{array}{l}\text { Knee flexion } \\
\text { PROM }\end{array}$ & $\begin{array}{l}\text { There was significant } \\
\text { improvement during knee } \\
\text { flexion PROM after intervention } \\
\text { among three groups. There was } \\
\text { no significant difference } \\
\text { between groups, but floss group } \\
\text { has improved knee PROM a lot } \\
\text { than the other group. }\end{array}$ \\
\hline $\begin{array}{l}\text { Galis and } \\
\text { Cooper [24] }\end{array}$ & Experimental & $\begin{array}{l}30 \text { healthy } \\
\text { participants (16 } \\
\text { males, } 14 \text { female) } \\
\text { randomly } \\
\text { assigned into: } \\
\text { control group } 10 \\
\text { FLOSS } \\
10 \\
100 \\
\text { FLOSS }_{200} \\
\text { group: } 10\end{array}$ & Calf & $\begin{array}{l}\text { Applied from above } 5 \mathrm{~cm} \\
\text { ankle joint to tibia } \\
\text { tuberosity with } 50 \% \text { tension } \\
\text { on right calf or left calf. } \\
\text { Control group (underwrap } \\
\text { pressure applied at, } 5 \mathrm{~mm} \\
\mathrm{Hg} \text { ) } \\
\text { FLOSS } 150 \text { group: } \\
\text { flossband } \\
\text { pressure applied at } 150 \\
\text { mmHg } \\
\text { FLOSS } 200 \text { group: } \\
\text { flossband } \\
\text { pressure applied at } 200 \\
\text { mmHg }\end{array}$ & $\begin{array}{l}\text { Pre-posttest } \\
\text { Applying Flossband or underwrap combined } \\
\text { active - ankle ROM PF and DF exercises, } 2 \\
\text { minutes, and then did } 20 \text { repetitions of calf raise } \\
\text { exercise. }\end{array}$ & $\begin{array}{l}\text { Ankle ROM DF, } \\
\text { PF } \\
\text { peak torque } \\
\text { power }\end{array}$ & $\begin{array}{l}\text { There was no significant } \\
\text { difference between groups } \\
\text { during these outcome measure. } \\
\text { There was significant difference } \\
\text { in FLOSS }{ }_{150} \text { group during } \\
\text { DFROM, but there was } \\
\text { improvement trend of DF power. }\end{array}$ \\
\hline $\begin{array}{l}\text { García-Luna } \\
\text { [7] }\end{array}$ & Pilot & $\begin{array}{l}5 \text { male athletes } \\
\text { (Age: } 20 \pm 0.5 \text { ) } \\
\text { with PFPS }\end{array}$ & Knee joint & $\begin{array}{l}\text { Life Flossband } \\
\text { Wrapping } \\
\text { flossband around the knee } \\
\text { joint from proximal to distal } \\
(182 \pm 38 \mathrm{mmHg}) \text {. }\end{array}$ & $\begin{array}{l}\text { Pre-posttest (wrapped flossband, remove } \\
\text { flossband immediately test) } \\
\text { Test would be executed into two days. In the } \\
\text { first day, all participants were accepted CMJ } \\
\text { (repeat three times and take the average) and } \\
\text { VAS test before formal study, then they were } \\
\text { wrapped by flossband around the knee and did } \\
\text { the same test, after that they were removed the } \\
\text { flossband and did the same test. In the second } \\
\text { day, all participants did the same test but without } \\
\text { applied flossabnd during three parts of testing. } \\
\text { 1-minute rest between series, and } 15 \text { seconds } \\
\text { between epetitions. }\end{array}$ & $\begin{array}{l}\text { VAS } \\
\text { CMJ (jump } \\
\text { height, jump } \\
\text { velocity, jump } \\
\text { power, jump } \\
\text { force) }\end{array}$ & $\begin{array}{l}\text { There was significant } \\
\text { improvement in all outcome } \\
\text { measure between pretest and } \\
\text { posttest. There was no } \\
\text { significant difference without } \\
\text { fossband during the second day } \\
\text { testing. }\end{array}$ \\
\hline
\end{tabular}


Table 2. Continued

\begin{tabular}{|c|c|c|c|c|c|c|c|}
\hline Kaneda [9] & Experimental & $\begin{array}{l}20 \text { healthy young } \\
\text { men (age: } 22.5 \pm \\
1.0 \text { ) }\end{array}$ & Calf & $\begin{array}{l}\text { Flossband } \\
\text { (Sanctband COMPRE Floss } \\
\text { TM Blueberry) } \\
\text { Wrapped around calf } \\
\text { muscles from above the } \\
\text { ankle to under knee joint. } \\
\text { (non-dominant leg, } 160 \pm 3 \\
\mathrm{mmHg} \text { ) }\end{array}$ & $\begin{array}{l}\text { Pre-posttest ( } 2 \text { time } * 3 \text { intervention) } \\
\text { All participants sited on the dynamometer of } \\
\text { Cybex with knee extension and ankle fixed on } \\
\text { the footplate, then they did DF by using Cybex } \\
\text { at a velocity of } 5 \% / \mathrm{s} \text { from GC-relaxed position } \\
\text { to maximum most DF position which } \\
\text { participants can most tolerate the GS stretch (I } \\
\text { minute hold, } 5 \text { repetations). } \\
\text { Then all participants rest } 6 \text { minutes on the } \\
\text { isokinetic machine. } \\
\text { After } 7 \text { days, all participants were wrapped } \\
\text { flossband around calf, and they were given four } \\
\text { times passive twisting the wrapped part and they } \\
\text { were allowed to do PF and AF movement, } 20 \\
\text { times. }\end{array}$ & $\begin{array}{l}\text { DFROM, passive } \\
\text { moment }\left(0^{\circ}, 20^{\circ}\right. \\
\text { and at the end of } \\
\text { ROM }) \text {, fascicle } \\
\text { length, muscle } \\
\text { hardness, MVC, } \\
\text { RFD }(0-50 \mathrm{~ms} \text {, } \\
0-100 \mathrm{~ms} \text {, } \\
0-150 \mathrm{~ms} \text { and } \\
0-200 \mathrm{~ms}) \text { and } \\
\text { muscle } \\
\text { activity }\end{array}$ & $\begin{array}{l}\text { There were significant changes } \\
\text { in RFD 0-50 and RFD } 0-100 \mathrm{~ms} \\
\text { between Flossing and SS. The } \\
\text { pre-post changes in DFROM } \\
\text { was significantly higher with } \\
\text { Flossing compared to Rest. }\end{array}$ \\
\hline Kaneda [25] & Crossover & $\begin{array}{l}17 \text { healthy young } \\
\text { men (Age: } 23.2 \pm \\
1.1) \\
\text { Floosband was } \\
\text { applied on } \\
\text { hamstring of } \\
\text { dominant leg }\end{array}$ & Thigh & $\begin{array}{l}\text { Flossband (Sanctband } \\
\text { COMPRE Floss Blueberry) } \\
\text { Wrapped around hamstring } \\
\text { muscles from above the } \\
\text { knee to under AIIS. }\end{array}$ & $\begin{array}{l}\text { Pre-posttest ( } 2 \text { time } * 3 \text { intervention) } \\
\text { All participants went through three intervention } \\
\text { pharse on three separate days. } \\
\text { Phrase 1: All participants were given passive } \\
\text { twisting and active knee flexion movement with } \\
\text { the lower leg tightly wrapped flossband. } \\
\text { Phase 2: They were given hamstring DS without } \\
\text { flossband in upright position, } 30 \text { secs stretch, } 4 \\
\text { sets, rest } 20 \text { secs } \\
\text { Phase 3: only did active knee flexion movement } \\
\text { without flossband. }\end{array}$ & $\begin{array}{l}\text { SLR test, } \\
\text { KE test, passive } \\
\text { torque, passive } \\
\text { stiffness, fascicle } \\
\text { length, maximal } \\
\text { isometric knee } \\
\text { flexion } \\
\text { contraction, } \\
\text { maximal } \\
\text { eccentric knee } \\
\text { extension/flexion } \\
\text { contraction, } \\
\text { RFD, and muscle } \\
\text { activity. }\end{array}$ & $\begin{array}{l}\text { There were significant } \\
\text { improvement in the SLR test, } \\
\text { passive KE test, passive torque at } \\
\text { end of ROM and maximal } \\
\text { eccentric knee flexion } \\
\text { contraction in flossband } \\
\text { comparing control intervention. }\end{array}$ \\
\hline Konrad [17] & Cross-over & $\begin{array}{l}16 \text { healthy males } \\
\text { (Age:25.69 } \pm 4.1 \text { ) }\end{array}$ & Thigh & $\begin{array}{l}\text { Flossand (Ludwig Artzt } \\
\text { GmbH, Dornburg, } \\
\text { Germany) } \\
\text { Wrapped } 4.5-5 \mathrm{~cm} \text { above } \\
\text { the patella until the } \\
\text { trochanter major (both } \\
\text { thing), } 50 \% \text { tension }\end{array}$ & $\begin{array}{l}\text { Pre-posttest } \\
\text { Two conditions were randomly tested in } \\
\text { different days (interval } 48 \mathrm{~h} \text { ) } \\
\text { Warm-up } 10 \text { minutes. } \\
\text { All participants were given } 2 \text { minutes flossband } \\
\text { treatment ( } 20 \text { squats), after } 48 \text { hours, they were } \\
\text { asked to do } 20 \text { squats without flossband. }\end{array}$ & $\begin{array}{l}\text { ROM (hip } \\
\text { flexion and knee } \\
\text { extensor), MVC, } \\
\text { PRT,CMJ }\end{array}$ & $\begin{array}{l}\text { There were only significant } \\
\text { effect on the MVC of the knee } \\
\text { extensors between flossband } \\
\text { condition and control condition. }\end{array}$ \\
\hline
\end{tabular}


Table 2. Continued

\begin{tabular}{|c|c|c|c|c|c|c|c|}
\hline Mills [13] & Cross-over & $\begin{array}{l}14 \text { professional } \\
\text { male } \\
\text { rugby union } \\
\text { athletes (Age: } \\
23.9 \pm 2.7 \text { ) }\end{array}$ & Ankle & $\begin{array}{l}\text { Flossband } \\
\text { (Life Flossband) } \\
\text { Applied flossband on both } \\
\text { ankles (wrapped by using } \\
\text { ankle tape method, } 180 \\
\text { mmHg) }\end{array}$ & $\begin{array}{l}\text { Pre-posttest (posttest was test after } 5 \text { minutes } \\
\text { and after } 30 \text { minutes) } \\
\text { All participants went through two different trials } \\
\text { interval } 7 \text { days), one is flossbans trial, and } \\
\text { another was control trial (without flossband) } \\
5 \text { minutes' warm-up exercises } \\
\text { Flossband session: all participants were asked to } \\
\text { seat on the floor with both knee full extension, } \\
\text { and they did full range of ankle PF and DF ( } \\
\text { minutes, } 2 \text { secs/ each). Flossband was removed, } \\
\text { and they were tested after } 5 \text { minutes and } 30 \\
\text { minutes. } \\
\text { Control session: all particpants did the same } \\
\text { exercise but without wrapping flossband. }\end{array}$ & $\begin{array}{l}\text { Ankle ROM, } \\
\text { CMJ, 5-m Sprint } \\
\text { (secs) } \\
10-\mathrm{m} \text { Sprint } \\
\text { (secs) } \\
15-\mathrm{m} \text { Sprint } \\
\text { (secs). } \\
20-\mathrm{m} \text { Sprint } \\
\text { (secs) }\end{array}$ & $\begin{array}{l}\text { There was no significant } \\
\text { differences between FLOSS and } \\
\text { CON for any outcome measures, } \\
\text { but there was small effect size } \\
\text { improvement trend for CMJ } \\
\text { performance 5minutes post and } \\
10-\mathrm{m} \text { Sprint, } 15-\mathrm{m} \text { Sprint after } \\
\text { flossband intervention. }\end{array}$ \\
\hline Pisz [26] & $\begin{array}{l}\text { Meta-analysis } \\
\text { and } \\
\text { Systematic } \\
\text { review }\end{array}$ & $\begin{array}{l}4 \text { articles were } \\
\text { included }\end{array}$ & Ankle/calf & $\begin{array}{l}\text { Flossband applied on calf/ } \\
\text { ankle }\end{array}$ & Pre-posttest & $\begin{array}{l}\text { Ankle ROM } \\
\text { CMJ }\end{array}$ & $\begin{array}{l}\text { There was a significant } \\
\text { improvement in ankle ROM } \\
\text { after flossband intervention, but } \\
\text { there were insufficient evidences } \\
\text { of flossband on jump } \\
\text { performance. }\end{array}$ \\
\hline Vogrin [16] & Crossover & $\begin{array}{l}19 \text { healthy } \\
\text { participants (14 } \\
\text { males, } 5 \text { females, } \\
\text { Age: } 23.78 \pm \\
4.85) \\
\text { Three conditions: } \\
\text { Condition 1: low } \\
\text { pressure } \\
\text { Condition 2: } \\
\text { moderate } \\
\text { pressure } \\
\text { Condition 3: high } \\
\text { pressure }\end{array}$ & Thigh & $\begin{array}{l}\text { Flossband } \\
\text { Wrapped on the dominant } \\
\text { thigh (50\%, from the above } \\
\text { the patella to below AIIS) }\end{array}$ & $\begin{array}{l}\text { Pre-posttest (posttest was immediate after } \\
\text { flossband and post } 30 \text { minutes after flossband) } \\
\text { All participants were given three similar } \\
\text { interventions (different degree of wrapping } \\
\text { pressure, low, moderate and high) at three } \\
\text { different visits ( } 48 \text { hours between each visit). } \\
\text { During each visit, pre and post-test data were } \\
\text { collected. } \\
\text { Low pressure }(<20 \mathrm{mmHg}) \\
\text { Moderate }(100-140 \mathrm{mmHg}) \\
\text { High }(150-210 \mathrm{mmHg}) \\
\text { All participants were asked to sit to the } \\
\text { therapeutic table and did knee extension and } \\
\text { flexion from } 90^{\circ} \text { to } 0^{\circ}, 2 \text { minutes, } 3 \text { sets, } 90 \text { secs } \\
\text { interval. }\end{array}$ & $\begin{array}{l}\text { ALSR, knee } \\
\text { extensor and } \\
\text { flexor MVC, } \\
\text { TcRF }(\mathrm{ms}), \\
\text { TcVM(ms), } \\
\text { TcBF }(\mathrm{ms}) \\
\operatorname{DmRF}(\mathrm{mm}) \\
\operatorname{DmVM}(\mathrm{mm}) \\
\operatorname{DmBF}(\mathrm{mm})\end{array}$ & $\begin{array}{l}\text { There was significant difference } \\
\text { for the low condition between } \\
\text { pre and posttest during the knee } \\
\text { extensor MVC } \text { (post } \\
\text { immediately and post } 30 \\
\text { minutes), TcRF (post } \\
\text { immediately) and TcVM (post } \\
30 \text { minutes). } \\
\text { There was no significant } \\
\text { difference between groups } \\
\text { among these outcomes, but there } \\
\text { were medium benefits between } \\
\text { low condition and high condition } \\
\text { (TcRF and knee extension } \\
\text { MVC), low condition and } \\
\text { control condition (DmRF), and } \\
\text { high condition to control } \\
\text { condition (DmBF). }\end{array}$ \\
\hline
\end{tabular}


Table 2. Continued

\begin{tabular}{|c|c|c|c|c|c|c|c|}
\hline Vogrin [27] & Crossover & $\begin{array}{l}30 \text { healthy } \\
\text { participants (18 } \\
\text { males, } 12 \\
\text { females, Age: } \\
23 \pm 4.51 \text { ) } \\
\text { Floss group: } \\
\text { Applied on the } \\
\text { random chosen } \\
\text { ankle (12 right } \\
\text { and } 18 \text { left) } \\
\text { Control: the other } \\
\text { ankle }\end{array}$ & Ankle & $\begin{array}{l}\text { Flossband (Medical } \\
\text { Flossing Band } 1.3 \mathrm{~mm} \text { ) } \\
\text { Standard figure } 8 \text { ankle tape } \\
\text { method }\end{array}$ & $\begin{array}{l}\text { Pre-posttest (post } 5 \text { post } 15 \text { post } 30 \text { post } 45 \text { ) } \\
\text { All participants did } 6 \text { minutes' warm-up, then } \\
\text { they did the immediate test as the baseline. } \\
\text { Random chosen the ankle and applied the } \\
\text { flossband, and then they were asked to do active } \\
\text { ankle PF and DF on both ankles with knee } \\
\text { extension sitting on bed ( } 2 \text { minutes/ set, } 3 \text { sets, } \\
\text { rest } 2 \text { minutes between each set), after complete } \\
\text { the ankle exercise, they were instructed to walk } \\
\text { around } 2 \text { minutes. The posttest conducted after } \\
5,15,30 \text { and } 45 \text { minutes' intervention. }\end{array}$ & $\begin{array}{l}\text { Active ankle PF } \\
\text { and DF ROM, }\end{array}$ & $\begin{array}{l}\text { There were significant } \\
\text { differences between baseline and } \\
\text { posttest (post5 post15 post30 } \\
\text { post45) in ankle DF ROM within } \\
\text { groups, only ankle PF ROM in } \\
\text { flossband group. }\end{array}$ \\
\hline $\begin{array}{l}\text { Kielur and } \\
\text { Powden }[8]\end{array}$ & $\begin{array}{l}\text { Meta-analysis } \\
\text { and } \\
\text { Systematic } \\
\text { review }\end{array}$ & $\begin{array}{l}6 \text { articles were } \\
\text { included }\end{array}$ & Ankle & $\begin{array}{l}\text { Flossband } \\
\text { Wrapped on ankle }\end{array}$ & $\begin{array}{l}6 \text { studies were included ( } 4 \text { high qualities, } 2 \text { low } \\
\text { quality) }\end{array}$ & Ankle DF ROM & $\begin{array}{l}\text { There was moderate } \\
\text { improvement PF ROM between } \\
\text { pre and posttest, but there was no } \\
\text { significant difference between } \\
\text { floss group and control group. }\end{array}$ \\
\hline $\begin{array}{l}\text { Quiles-Sanc } \\
\text { hez [10] }\end{array}$ & $\begin{array}{l}\text { Systematic } \\
\text { review }\end{array}$ & $\begin{array}{l}9 \text { articles were } \\
\text { enrolled }\end{array}$ & - & - & - & $\begin{array}{l}\text { Strength, } \\
\text { endurance and } \\
\text { function }\end{array}$ & $\begin{array}{l}\text { Flossband is an effective method } \\
\text { on strength, edurance and } \\
\text { physical function. }\end{array}$ \\
\hline $\begin{array}{l}\text { Hodeaux } \\
{[28]}\end{array}$ & Crossover & $\begin{array}{l}12 \text { healthy elite } \\
\text { tennis players (6 } \\
\text { males and } 6 \\
\text { females, age: } 20.5 \\
\pm 1.24 \text { ) } \\
\text { Random assign } \\
\text { into two separate } \\
\text { sessions (interval } \\
24 \text { hours) }\end{array}$ & Elbow & $\begin{array}{l}\text { Voodoo Flossband (50\% } \\
\text { tension) } \\
\text { Wrapped around elbow } \\
\text { joint }\end{array}$ & $\begin{array}{l}\text { Flossband session (pre-posttest)Applied } \\
\text { flossband on the elbow and did } 6 \text { passive } \\
\text { exercises within } 2 \text { minutes ( } 3 \text { repetation/ } \\
\text { exercise) } \\
\text { Control session: did the same exercises without } \\
\text { flossband. }\end{array}$ & $\begin{array}{l}\text { Passive elbow } \\
\text { with extension } \\
\text { and flexion } \\
\text { ROM, forearm } \\
\text { pronation and } \\
\text { supination }\end{array}$ & $\begin{array}{l}\text { There was no significant } \\
\text { difference between groups } \\
\text { during these parameters } \\
\text { measure, but there was } \\
\text { improvement trend for elbow } \\
\text { ROM in flossband session } \\
\text { comparing with without } \\
\text { flossband session. }\end{array}$ \\
\hline $\begin{array}{l}\text { McCallum } \\
{[29]}\end{array}$ & Experimental & $\begin{array}{l}30 \text { ankle } \\
\text { inversion } \\
\text { participants (age: } \\
18 \text { to } 45 \text { ) } \\
\text { Random assigned } \\
\text { into three groups }\end{array}$ & $\begin{array}{l}\text { chronic } \\
\text { ankle } \\
\text { inversion } \\
\text { injury }\end{array}$ & $\begin{array}{l}\text { Flossband (50\% tension) } \\
\text { Applied standard figure } 8 \\
\text { ankle tape }\end{array}$ & $\begin{array}{l}\text { Pre-posttest }(5 \text { minutes after treatment in the } \\
\text { fourth time and } 7 \text { times) } \\
\text { Group } 1 \text { chiropractic manipulation combined } \\
\text { with flossband } \\
\text { Group2: flossband ( } 20 \text { repetition ankle active PF } \\
\text { AND DF within } 2 \text { minutes) } \\
\text { Group3: chiropractic manipulation (Anterior to } \\
\text { posterior) } 7 \text { time/ } 3 \text { weeks }\end{array}$ & $\begin{array}{l}\text { WBLT, ankle PF, } \\
\text { and DF ROM }\end{array}$ & $\begin{array}{l}\text { There was a significant } \\
\text { difference among the three } \\
\text { groups after } 4^{\text {th }} \text { intervention and } \\
7^{\text {th }} \text { during EBL PF ROM and DF } \\
\text { ROM, but there was no } \\
\text { significant difference between } \\
\text { the } 4^{\text {th }} \text { intervention and } 7^{\text {th }} \\
\text { intervention and among groups. }\end{array}$ \\
\hline
\end{tabular}


Table 2. Continued

\begin{tabular}{|c|c|c|c|c|c|c|c|}
\hline Maust [12] & Crossover & $\begin{array}{l}21 \text { healthy active } \\
\text { participants }(8 \\
\text { males, } 13 \\
\text { females, age: } \\
22.62 \pm 2.99) \\
\text { Floss group, sham } \\
\text { group, and } \\
\text { control group }\end{array}$ & Thigh & $\begin{array}{l}\text { Voodoo flossband ( } 50 \% \\
\text { tension) } \\
\text { Wrapped from above the } \\
\text { patella and ending at the } \\
\text { gluteal fold. }\end{array}$ & $\begin{array}{l}\text { Pre-posttest } \\
\text { Three groups did } 10 \text { bodyweight squats; } 10 \\
\text { lunges and } 20 \text { standing hamstring curls with } \\
\text { flossband, sham, and without flossband } \\
\text { separately. }\end{array}$ & $\begin{array}{l}\text { Hip Flexion } \\
\text { ROM } \\
\text { CMJ }\end{array}$ & $\begin{array}{l}\text { There was significant difference } \\
\text { among three groups in hip ROM, } \\
\text { and there was also significant } \\
\text { differences within flossband } \\
\text { group and sham group in hip } \\
\text { ROM. }\end{array}$ \\
\hline $\begin{array}{l}\text { Hadamus } \\
{[14]}\end{array}$ & Experimental & $\begin{array}{l}40 \text { healthy } \\
\text { athletes } \\
\text { Randomize } \\
\text { assigned into } 2 \\
\text { groups } \\
\text { Floss group } \\
\text { Control group }\end{array}$ & Thigh & $\begin{array}{l}\text { Voodoo flossband ( } 50 \% \\
\text { tension) } \\
\text { Wrapped from above the } \\
\text { patella to the end of gluteal } \\
\text { fold. }\end{array}$ & $\begin{array}{l}\text { Pre-posttest (posttest was tested on immediately } \\
\text { test, } 15 \text { minutes, } 30 \text { minutes and } 45 \text { minutes) } \\
\text { Warm-up lasts } 9 \text { minutes }\end{array}$ & Sit and reach test & $\begin{array}{l}\text { There was a significant } \\
\text { difference between the floss } \\
\text { group and the control group after } \\
30 \text { minutes of intervention, } \\
\text { however, there was an increasing } \\
\text { trend comparing floss group to } \\
\text { control group in different } \\
\text { posttest. }\end{array}$ \\
\hline
\end{tabular}

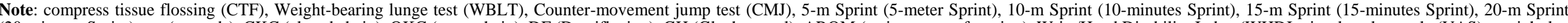

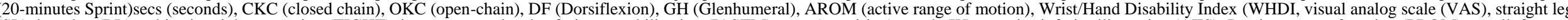

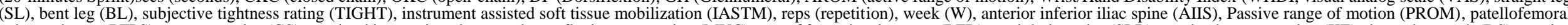

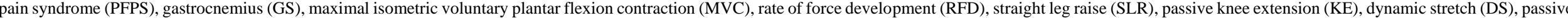

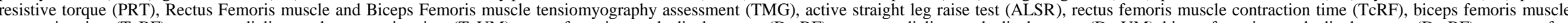

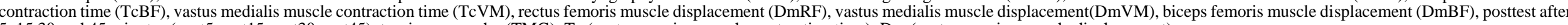
$5,15,30$ and 45 minutes (post5 post15 post30 post45), tensiomypgraphy (TMG), Tc (gastrocnemius muscle contraction time), Dm (gastrocnemius muscle displacement) 
Table 3. Flossing band application and outcomes measures

\begin{tabular}{|c|c|c|c|c|c|c|c|c|c|c|c|c|c|c|}
\hline \multirow[b]{2}{*}{ No. } & \multirow[b]{2}{*}{ Author } & \multirow[b]{2}{*}{ Country } & \multirow[b]{2}{*}{ Participant } & \multirow{2}{*}{$\begin{array}{l}\text { Intervention } \\
\text { period }\end{array}$} & \multirow{2}{*}{$\begin{array}{l}\text { Wrapping } \\
\text { Body part }\end{array}$} & \multirow{2}{*}{$\begin{array}{c}\text { Flossband } \\
\text { Tension }\end{array}$} & \multicolumn{8}{|c|}{ Outcome Measures } \\
\hline & & & & & & & ROM & VAS & $\begin{array}{c}\text { Muscle } \\
\text { tightness }\end{array}$ & Strength & Squat & $\begin{array}{l}\text { Muscle } \\
\text { activity }\end{array}$ & Sprint & CMJ \\
\hline 1 & Driller [19] & $\begin{array}{c}\text { New } \\
\text { Zealand }\end{array}$ & $\begin{array}{l}\text { Healthy } \\
\text { athletes }\end{array}$ & Acute effect & Ankle & $178 \pm 18 \mathrm{mmHg}$ & $\sqrt{ }$ & & & & & & $\sqrt{ }$ & $\sqrt{ }$ \\
\hline 2 & $\begin{array}{l}\text { Driller and Overmaye } \\
{[15]}\end{array}$ & $\begin{array}{c}\text { New } \\
\text { Zealand }\end{array}$ & $\begin{array}{l}\text { Healthy } \\
\text { athletes }\end{array}$ & Acute effect & Ankle & $178 \pm 18 \mathrm{mmHg}$ & $\sqrt{ }$ & & & & & & & $\sqrt{ }$ \\
\hline 3 & Hagen [11] & - & $\begin{array}{l}\text { Healthy } \\
\text { college } \\
\text { student }\end{array}$ & Acute effect & Ankle & - & $\sqrt{ }$ & & & & $\sqrt{ }$ & & & \\
\hline 4 & Kiefer [20] & USA & $\begin{array}{l}\text { Healthy } \\
\text { college } \\
\text { student }\end{array}$ & Acute effect & Arm & $50 \%$ tension & $\sqrt{ }$ & & & & & & & \\
\hline 5 & $\begin{array}{l}\text { Ross and Kandassamy } \\
{[21]}\end{array}$ & UK & $\begin{array}{c}\text { Heaithy } \\
\text { participant }\end{array}$ & Acute effect & $\begin{array}{l}\text { Achilles } \\
\text { tendon }\end{array}$ & $50 \%$ tension & $\sqrt{ }$ & & & & & & & \\
\hline 6 & Stevenson [22] & USA & Patient & Acute effect & Ankle & $180 \mathrm{mmHg}$ & $\sqrt{ }$ & & & & & & & \\
\hline 7 & Williams [23] & USA & $\begin{array}{c}\text { Healthy } \\
\text { participants }\end{array}$ & 4 weeks & Ankle & - & $\sqrt{ }$ & & & & & & & \\
\hline 8 & Cheatham [5] & USA & $\begin{array}{c}\text { Healthy } \\
\text { participants }\end{array}$ & Acute effect & Thigh & $50 \%$ tension & $\sqrt{ }$ & & & & & & & \\
\hline 9 & Galis and Cooper [24] & UK & $\begin{array}{c}\text { Healthy } \\
\text { participants }\end{array}$ & Acute effect & Calf & $\begin{array}{l}<20 \mathrm{mmHg} \\
150 \mathrm{mmHg} \\
200 \mathrm{mmHg}\end{array}$ & $\sqrt{ }$ & & & $\sqrt{ }$ & & & & \\
\hline 10 & García-Luna [7] & Spain & Patient & Acute effect & Knee & $182 \pm 38 \mathrm{mmHg}$ & & $\sqrt{ }$ & & & & & & $\sqrt{ }$ \\
\hline 11 & Kaneda [25] & Japan & $\begin{array}{c}\text { Healthy } \\
\text { participants }\end{array}$ & Acute effect & Calf & $160 \pm 3 \mathrm{mmHg}$ & $\sqrt{ }$ & & & $\sqrt{ }$ & & $\sqrt{ }$ & & \\
\hline 12 & Kaneda [9] & Japan & $\begin{array}{c}\text { Healthy } \\
\text { participants }\end{array}$ & Acute effect & Thigh & - & & & $\sqrt{ }$ & $\sqrt{ }$ & & $\sqrt{ }$ & & \\
\hline 13 & Konrad [17] & Austria & $\begin{array}{c}\text { Healthy } \\
\text { participants }\end{array}$ & Acute effect & Thigh & $50 \%$ tension & $\sqrt{ }$ & & & $\sqrt{ }$ & & $\sqrt{ }$ & & $\sqrt{ }$ \\
\hline 14 & Mills [13] & $\begin{array}{c}\text { New } \\
\text { Zealand }\end{array}$ & $\begin{array}{l}\text { Healthy } \\
\text { athletes }\end{array}$ & Acute effect & Ankle & $180 \mathrm{mmHg}$ & $\sqrt{ }$ & & & & & & $\sqrt{ }$ & $\sqrt{ }$ \\
\hline 15 & Vogrin [27] & Slovenia & $\begin{array}{c}\text { Healthy } \\
\text { participants }\end{array}$ & Acute effect & Thigh & $\begin{array}{l}\text { Low }(<20 \\
\mathrm{mmHg}) \\
\text { Moderate }(100- \\
140 \mathrm{mmHg}) \\
\mathrm{High}>150 \\
\mathrm{mmHg}\end{array}$ & & & $\sqrt{ }$ & $\sqrt{ }$ & & $\sqrt{ }$ & & \\
\hline 16 & Vogrin [16] & Slovenia & $\begin{array}{c}\text { Healthy } \\
\text { participants }\end{array}$ & Acute effect & Ankle & - & $\sqrt{ }$ & & & & & & & \\
\hline
\end{tabular}


The Effect of Tissue Flossing Technique on Sports and Injury Prevention and Rehabilitation: A Systematic Review of Recent Research

Table 3. Continued

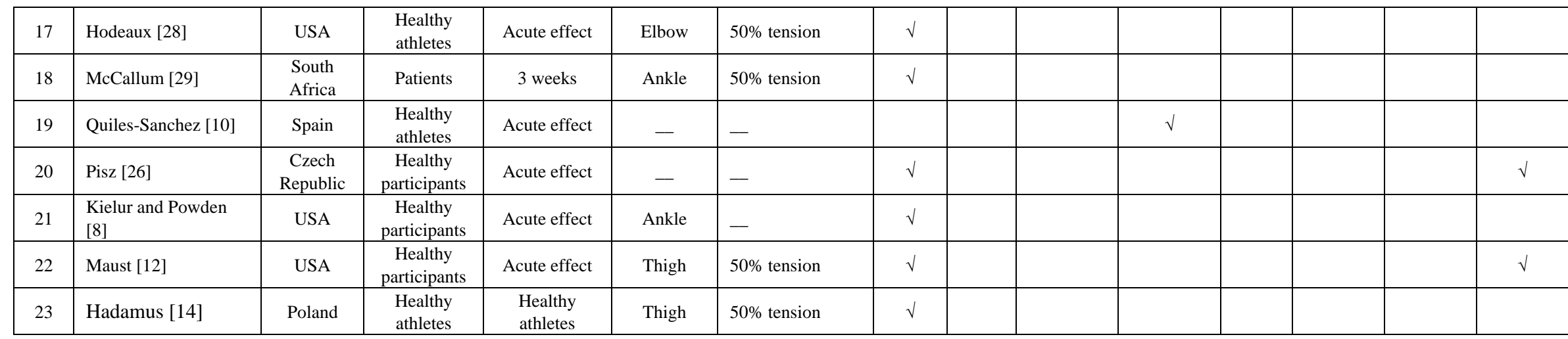

Note: Counter-movement jump test (CMJ), range of motion (ROM), visual analog scale (VAS). 
According to the data extracted in this review, it can be assumed that the tissue flossing technique has a positive effect on joint ROM, pain, muscle tightness, muscle strength, and physical function performance (see Table 3 ).

\subsection{The Effects of Flossing on Joint Range of Motion}

Nineteen out of 23 studies examined the effectiveness of flossband application on the ROM of various joints. Fourteen out of 19 individual studies focused on comparing the effects of flossband application on ankle dorsiflexion of range of motion (DFROM) to other interventions. The findings of these 14 studies show that there was no significant difference during DFROM between the flossband group and the control group, but there was a positive trend $[19,24]$. However, there was a significant difference of DFROM between the pre- and post-tests in the flossband group [15,20]. Systematic evidence showed that flossband treatment is a useful method to acutely increase the effects of ankle ROM, compared to other methods [30]. Only one out of 19 individual studies wrapped flossband on the upper arm, and the results showed that shoulder flexion was significantly improved [20]. In addition, four out of 19 individual studies proved that flossband application on the thigh could improve knee flexion ROM and hip flexion ROM. Meanwhile, Cheatham, [5] pointed that flossband intervention is more effective than soft tissue mobilization or other types of instruments in the aspect of joint flexibility.

\subsection{The Effects of Flossing on Pain}

Only 1 out of 23 studies investigated the effectiveness of the application of flossband on chronic pain management. In García-Luna's [7] pilot study, five young male recreational athletes diagnosed with chronic patellarfemoral pain syndrome (PFPS) were recruited, and all the participants were instructed to do three counter movement jumps (CMJs) with a flossband wrapping above the patellar (with $182 \pm 38 \mathrm{mmHg}$ ), or without it, during pre- and post-tests for evaluation of the acute effect of variation of knee pain intensity. The results showed that the application of flossband on patients with chronic PFPS was found to significantly decrease the perception of knee pain (Analogue visual scale, VAS) after immediately removing the flossband [7]. However, there was no significant difference in a variety of pain perceptions on the affected knee between the flossband group and the control group.

\subsection{The Effects of Flossing on Muscle Tightness}

Two out of 23 studies examined the acute effect of flossband application on hamstring tightness by comparing the straight leg raise test (SLR). A similar flossband wrapping method, the application on the dominant thigh of healthy participants, was used in these two studies, but different interventions were compared in the aspect of effectiveness of flossband application on muscle tightness. One finding revealed that there was no significant difference in hamstring tightness among the groups (control group with a pressure of less than 20 $\mathrm{mmHg}$, a moderate group with $100 \mathrm{mmHg}$ to $140 \mathrm{mmHg}$, and high group with $150 \mathrm{mmHg}$ to $210 \mathrm{mmHg}$ ), and within the groups (pre, post immediate and post 30 minutes). However, the small to medium improvement of SLR was found between the group with moderate pressure and the control group with low pressure. Small benefits were associated between the application of the flossband with the high pressure and low pressure, and there was a beneficial trend compared to the groups with low and high pressure respectively [27]. Another result showed that there was a significant difference in SLR changes after immediate removal of the flossband compared to the control group. In addition, the flossband as an effective instrument has the same benefit as dynamic stretching in reducing tightness of the hamstring, or it is even more beneficial [25].

\subsection{The Effects of Flossing on Muscle Strength}

Six out of 23 studies compared the acute effect of flossband application on the calf or thigh of healthy participants to improve ankle or knee strength, as well as muscle activation. Four out of 6 individual studies not only reported the effect of flossband application on strength, but it also associated on muscle activation. There was no significant difference of isometric maximal voluntary contraction (IMVC) of ankle plantar flexion force and gastrocnemius (GC) activation among the groups (group with flossband, group with static stretching, and group with resting), but the flossband application on the GC helps improve ankle dorsiflexion rate of force development for the first 100 seconds, compared to the control groups [25]. In addition, flossband application on the thigh has benefits in improving the IMVC of knee extension strength, or eccentric MVC of knee flexion, and increasing neuromuscular activity, compared to the control group [17,25,27]. Two out of 6 individual studies only mentioned flossband application on strength. In Galis' study [24], the result showed that there was no significant difference between the strength of ankle flexion and extension among the groups (control group, group with $150 \mathrm{mmHg}$ flossband wrapping pressure and $200 \mathrm{mmHg}$ flossband wrapping pressure), but flossband wrapping on the calf muscle with $150 \mathrm{mmHg}$ might be beneficial to improve ankle dorsiflexion peak torque (DFPT), compared to the control group and the group with high wrapping pressure [24]. DFPT was improved by up to $22 \%$ after flossband application [10]. Thus, flossband seems to be beneficial to enhance muscle strength. 


\subsection{The Effects of Flossing on Physical Function Performance}

Ten out of 23 studies examined the acute effect of flossband application on the squat, running, and jumping. Only one out of 10 individual studies compared different interventions on the performance of the body overhead weight squat. The study revealed that squat function was significantly improved after flossband application, but there was no significant difference in squat performance among the groups. Therefore, this result indicated that the flossband seems to be as effective as dynamic stretching with closed-kinematics chain or static stretching with closed-kinematics chain [11]. There are two out of 10 individual studies that compared the time of the 5-meter, 10-meter, and 15-meter sprint performance in healthy athletes between the flossband group and the control group. The results showed that there was an improvement in the 10-meter sprint and 15-meter sprint group compared to the control group, and these benefits will last up to 45 minutes [13,19,31]. Seven out of 10 studies compared the changes in jumping performance between the flossband group and the control group. Five out of 7 studies showed that there was a significant improvement in jump height, jump velocity, jump power, and jump force after applying a flossband. However, there was only an improvement trend between groups [7]. Only two studies presented that there was insufficient evidence about flossband improving jump performance [12]. Therefore, flossband could be a novel method to improve physical function.

\section{Discussion}

Flossband as a therapeutic intervention instrument could be used to increase ankle dorsiflexion ROM over the short term and the long term. Furthermore, small to large effect sizes have been found in previous studies. In the field of acute effects of flossband application on the ankle, Driller [19] found that flossband wrapped around the ankle significantly improved ankle dorsiflexion ROM in healthy young athletes. However, these two results were only associated with small effect sizes of 0.21 and 0.31 , respectively. In the same year, Hagen's study [11] showed similar evidence about flossband significantly improving ankle dorsiflexion ROM in healthy college students $(d=0.36)$. In the year 2021 , a systematic literature review and meta-analysis evidence discovered that ankle dorsiflexion ROM significantly increased after flossband intervention compared to the baseline, and the effect size was 0.46 [8]. At the level of equivalent evidence, only one study examined application flossband for 4 weeks, and the small effect size evidence showed that ankle dorsiflexion ROM was significantly increased after 4 weeks of intervention [23]. In contrast to the above studies, Galis and Cooper [24], Kaneda [25], and Ross and Kandassamy [21] pointed out that flossband significantly increased ankle dorsiflexion ROM with medium effect size in healthy active young participants, and the effect size was $0.77,0.61,0.56$, respectively. Vogrin [27] also showed that flossband intervention significantly increased ankle dorsiflexion ROM with medium to large effect size in healthy young participants. McCallum's [29] study revealed that 3 weeks of applying flossband on patients with chronic ankle sprains also significantly improved ankle dorsiflexion ROM with medium effect size $(d=0.76)$. The varying results on different levels of improvement after flossband application were due to the amount of tension or wrapping pressure and wrappling methods in these studies. Around $50 \%$ tension of flossband or $150 \mathrm{mmHg}$ wrapping pressure could improve ankle dorsiflexion ROM more than the other types of wrapping tension and pressure. In Galis and Cooper's [24] study, the researcher found that applying $150 \mathrm{mmHg}$ wrapping pressure on the ankle could significantly increase dorsiflexion ROM compare to $200 \mathrm{mmHg}$ wrapping pressure and low wrapping pressure. Kaneda [25], Ross and Kandassamy [21], and McCallum [29] also pointed out that $160 \pm 3 \mathrm{mmHg}$ or $50 \%$ tension could improve ROM with medium effect size compared with the other studies which used high pressure or stretch tension. Meanwhile, the variance of flossband application on different parts of the human body could be another factor that led to the differing results. Therefore, $50 \%$ tension of flossband or $150 \mathrm{mmHg}$ wrapping pressure could be a generalization for the other joints and more research about the long-term effects of flossband should be conducted in future studies.

Flossband application is not only beneficial to improve ankle ROM, but has also been proven to increase the ROM of other joints. Kiefer assessed the effect of flossband on the shoulder joint of healthy college students, and the results showed that the acute intervention significantly increased shoulder flexion ROM [20]. Hadamus [14] concluded that trunk flexibility was also significantly improved after using flossband as a warm-up assistant tool compared to the control group. Knee flexion ROM was significantly improved after flossband application, but this improvement only had a small effect size $(\mathrm{d}=0.47)$ [5]. Maustk found that flossband can be used to increase hip flexion when compared with to sham group or control group [12], but Konrad [17] mentioned that flossband may not be used to increase hip flexion. Hodeaux [28] pointed out that there was no significance for elbow flexion ROM between the flossband group and control group, but there was an improvement trend with small effect size $(\mathrm{d}=0.32)$. By reviewing the studies of Hodeaux [28] and Konrad [17], small sample size may be one of the factors which led to a significant effect. Therefore, future studies should aim to recruit a bigger sample size. In addition, it is worth noting that most studies were focused on applying flossband on the ankle joint, especially on healthy athletes or active young people 
rather than patients. Therefore, there was less evidence showing the effects of flossband on the patients as well as the effect of flossband application on other body parts or joints. Thus, more related evidences should be sought after in future studies.

Flossband seems to act as an effective pain management tool by producing shear force on myofascia, increasing joint space, and improving blood circulation. That said, there was only one article reporting the application of flossband on knee pain patients. After flossband application, perceived pain in the knee was significantly reduced, and this study showed a big effect size [7]. Interestingly, the available evidence of flossband on pain management was scarce. Thus, more studies about the effect of flossband application for pain management of different etiologies should be conducted in the future.

Flossband might be used into reducing muscle tightness, thus, flossband could be a potentially therapeutic tool for injury prevention and rehabilitation of the skeletal muscular fascial system disease. Applying flossband on the hamstring significantly changed its function, especially increasing hamstring flexibility. There were only two studies on muscle flexibility changes after applying flossband on soft tissues. All the participants were asked to do active knee flexion and knee extension exercises in 2 minutes by wrapping flossband on the hamstring, and the small to medium effect size evidence showed that straight leg raise test was improved after removing the flossband compared to the control group with moderate pressure of flossband application [27]. Kaneda [25] also supported that flossband wrapping around hamstring muscles from above the knee significantly enhanced straight leg raise performance compared to the group without flossband. The mechanism behind the muscle flexibility changes caused by flossband may be due to shear force during tissue flossing or the thermal effect during flossing. However, the exact physiological mechanism of flossband has not been examined. In the future, more studies about the effects of flossband on skeletal muscular fascial system and its physiological mechanism needs to be conducted.

Flossband is beneficial to enhance muscle strength, but there was controversial evidence of increasing muscle activity. Galis and Cooper [24] highlighted that nearly 150 $\mathrm{mmHg}$ of flossband wrapping pressure was possibly beneficial to improve ankle dorsiflexion peak torque after applying flossband on the calf muscles $(\mathrm{d}=0.27)$. However, over wrapping pressure was observed to impair ankle dorsiflexion peak torque ability (Galis \& Cooper, 2020). It can be seen that appropriate wrapping pressure may be an important factor affecting strength. Research finding showed that flossband wrapping around calf muscles significantly improved isometric maximal voluntary contraction (MVC) of ankle plantar flexion force at the end of plantar flexion $(\mathrm{d}=0.27)$ and enhanced rate of force development within $0-50 \mathrm{~ms}(\mathrm{~d}=0.38)$. Meanwhile, there was a trend to activate calf muscles $(\mathrm{d}=$
0.47) [9]. In contrast, evidence from a tensiomyography perspective showed that ankle flossband application on gastrocnemius muscle contraction time was not obvious [27]. The other evidences showed that applying flossband around the thigh improved eccentric MVC of the knee extension force $(d=0.59)$, flexion force $(d=0.56)$ and knee extension passive torque at the end of knee extension $(d=0.73)$ [25]. Konrad mentioned that wrapping flossband around the thigh significantly improved MVC of knee extension compared to the control group ( $\mathrm{d}=$ 0.77 ), but leg muscle activation was not found in this study [32]. Vogrin [16] and Vogrin [27] pointed out that wrapping flossband around the thigh provided small to medium benefits to improve isometric MVC of knee extension force compared to the control group, and it significantly increased isometric MVC of knee extension and rectus femoris muscle contraction time after flossing. The underlying physiological mechanisms of flossband on the aspect of force production and muscle activation have yet to be determined, but available evidences showed that there are several viewpoints of using flossband to improve force production mechanism as well as improve neuromuscular function. Flossband could facilitate fascial layers sliding or arrange the disordered fascial tissue. The smooth sliding between the layers of fluid fascia could mediate local muscle contraction or elongation as well as restoration of muscle length for optimum myosin head coupling mechanism [33]. The heat from intramuscular friction would be another important factor which could impact muscle contraction strength [9]. Soft tissue flossing with compression also is a critical factor which directed neuromuscular control of coordination and movement [33]. Some studies even suggested that tissue flossing might promote the release of growth hormones and norepinephrine level following the compression [32]. Flossband wrapping on the soft tissue indeed increased muscle strength and enhanced rate of force development, but it seemed to be not present in neuromuscular muscle activation. Therefore, essential evidence by using surface EMG evidence, ultrasound image evidence and physiological evidence on the level of neurological function after flossing needs to be investigated in the future.

Human functional movement quality can be improved through the tissue flossing technique. Flossband wrapping around ankle significantly improved squat performance when compared to pre-test performance, and it provided medium effect size benefits [11]. Small effect size evidence showed that wrapping flossband around the ankle could be beneficial for sprint performance and vertical jump ability compared to the control group [19]. Another evidence supported that there was small effect size enhancement in jump height and jump velocity between the flossband group and control group, and there was significant improvement in jump height after flossing [19]. Applying flossband on the knee pain patients also improved jump performance. This result was consistent with the previous findings, but the difference was that it 
also improved jump velocity, jump power, jump force and jump time in this study. Therefore, the use of flossband on patients led to obvious improvements in jumping performance [7]. Knorad [32] showed that flossband wrapping around the thigh could not increase the vertical jump height, and he also pointed out that flossband application on the thigh in healthy participants was not enough to induce significant changes in jump height because jumping is a complex function movement which is related to several muscle groups. Milles [13] found that applying flossband on the highly treated ruby athletes had less potential to improve jump performance and sprint performance. However, a meta-analyses evidence confirmed that there was a positive impact of flossing on jump performance [26]. Available evidence showed that the effects of flossband could lead to noticeable improvement in the general population or patients. Thus, more studies on different populations should be done in the future.

\section{Conclusions}

Flossband as a novel and emerging therapeutic tool has been used in sport as well as clinical practice in recent years. Based on the findings in previous studies, flossband wrapping on different soft tissues or peripheral joints could be a valid method to increase joint ROM, manage pain, and reduce muscle tightness. Not only that, it could also have other potential benefits and enhance muscle strength, exert higher rate of force development in per unit time, as well as improve physical functional performance such as squat, sprint and jump ability. In addition, the key to apply this technique is mastering the wrapping tension and pressure. Specifically, $50 \%$ flossing tension or approximately $150 \mathrm{mmHg}$ wrapping pressure could lead to positive effects on the outcomes of ROM, pain, muscle stiffness, muscle strength and physical function performance when compared with low or high tension or pressure. Therefore, the above findings indicate that flossband is a useful treatment option, and it can provide significant contribution in the field of athletic training, sports injury prevention and rehabilitation in the future.

\section{Perspective for Future Research}

Based on the previous studies, limited evidences showed that flossband as a novel technique provided small to medium effect size benefits in the aspects of increasing ROM, strengthening muscle force, releasing muscle tension and improving physical functional performance. Additionally, it showed a high effect size for pain management. Unfortunately, these evidences of flossband mostly involved soft tissues and joints of the limbs, and none of studies examined the effects on trunk function or evaluated its specific physiological mechanism. Therefore, it would be pertinent for future studies to broaden the field of flossband application and further investigate its physiological mechanism. In addition, most studies were mainly conducted on a population of healthy and active participants or well-trained athletes, and very little research has been done with patients. At the same time, those studies only investigated the short-term effects rather than the long-term effects of flossband application on different soft tissues or peripheral joints. Therefore, more studies about the effects of flossband application with patients should be implemented in the future, especially with regards to the long-term effects of flossband application.

\section{REFERENCES}

[1] Wilke, J.; Vleeming, A.; Wearing, S. Overuse Injury: The Result of Pathologically Altered Myofascial Force Transmission? Exercise and sport sciences reviews 2019, 47, 230-236.

[2] Angelopoulos, P.; Mylonas, K.; Tsepis, E.; Billis, E.; Vaitsis, N.; Fousekis, K. The Effects of Instrument-Assisted Soft Tissue Mobilization, Tissue Flossing, and Kinesiology Taping on Shoulder Functional Capacities in Amateur Athletes. Journal of Sport Rehabilitation 2021, 1, 1-10.

[3] Aicale, R.; Tarantino, D.; Maffulli, N. Overuse Injuries in Sport: A Comprehensive Overview. Journal of orthopaedic surgery and research 2018, 13, 1-11.

[4] Al - Haliq, M. The Effect of a Training Program Using Speed-Specific Strength Exercises on Sensory-Motor Perception in Learning Stroke Forehand Smash in Badminton Material for Students of the Hashemite University. International Journal of Human Movement and Sports Sciences 2020, 8, 299-307.

[5] Cheatham, S.; Martinez, R.; Montalvo, A.; Odai, M.; Echeverry, S.; Robinson, B.; Bailum, E.; Viecco, K. Comparison of Roller Massage, Instrument Assisted Soft-Tissue Mobilization, and Floss Band on Passive Knee Motion Among Inexperienced Individuals. Clinical Practice in Athletic Training 2020, 3, 24-36.

[6] Lim, W. Effects of Progressive Increased Intensity of PNF Stretching on Hamstring Force and Flexibility. International Journal of Human Movement and Sports Sciences 2020, 8, 186-192.

[7] García-Luna, M.A.; Cortell-Tormo, J.M.; González-Martínez, J.; García-Jaén, M. The Effects of Tissue Flossing on Perceived Knee Pain and Jump Performance: A Pilot Study. International Journal of Human Movement and Sports Sciences 2020, 8, 63-68.

[8] Kielur, D.S.; Powden, C.J. Changes of Ankle Dorsiflexion Using Compression Tissue Flossing: A Systematic Review and Meta-Analysis. Journal of Sport Rehabilitation 2020, 30, 306-314.

[9] Kaneda, H.; Takahira, N.; Tsuda, K.; Tozaki, K.; Sakai, K.; Kudo, S.; Takahashi, Y.; Sasaki, S.; Fukushima, K.; 
Kenmoku, T. The Effects of Tissue Flossing and Static Stretching on Gastrocnemius Exertion and Flexibility. Isokinetics and Exercise Science 2020, 28, 205-213.

[10] Quiles-Sanchez, L.V.; Baroutas, I.; Kyriakos, G.; Gravvanis, N.; Georgakopoulou, V.E.; Trakas, N.; Damaskos, C.; Garmpi, A.; Garmpis, N.; Antoniou, V. Medical Flossing and the Pilates Method: Their Effectiveness on the Strength, Endurance, and Functionality of Healthy Individuals. Cureus 2021, 13, 1-6.

[11] Hagen, N.; Reyes, C.; Liu, E. Can Compression Assist with Ankle Movement Preparation? A Comparison of Static, Dynamic, and Dynamic with Compression Stretching on Ankle Dorsiflexion Motion. Journal of Australian Strength and Conditioning 2017, 25, 6-17.

[12] Maust, Z.; Bradney, D.; Collins, S.M.; Wesley, C.; Bowman, T.G. The Effects of Soft Tissue Flossing on Hamstring Range of Motion and Lower Extremity Power. International Journal of Sports Physical Therapy 2021, 16, 689-694.

[13] Mills, B.; Mayo, B.; Tavares, F.; Driller, M. The Effect of Tissue Flossing on Ankle Range of Motion, Jump, and Sprint Performance in Elite Rugby Union Athletes. Journal of sport rehabilitation 2020, 29, 282-286.

[14] Hadamus, A.; Kowalska, M.; KĘdra, M.; Wiaderna, K.; Białoszewski, D. Effect of Hamstring Tissue Flossing during Warm-up on Sit and Reach Performance. The Journal of Sports Medicine and Physical Fitness 2021.

[15] Driller, M.W.; Overmayer, R.G. The Effects of Tissue Flossing on Ankle Range of Motion and Jump Performance. Physical Therapy in Sport 2017, 25, 20-24.

[16] Vogrin, M.; Kalc, M.; Ličen, T. Acute Effects of Tissue Flossing around the Upper Thigh on Neuromuscular Performance: A Study Using Different Degrees of Wrapping Pressure. Journal of Sport Rehabilitation 2020, 30, 601-608.

[17] Konrad, A.; Močnik, R.; Nakamura, M. Effects of Tissue Flossing on the Healthy and Impaired Musculoskeletal System: A Scoping Review. Frontiers in Physiology 2021, 12, 1-13.

[18] Shea, B.J.; Grimshaw, J.M.; Wells, G.A.; Boers, M.; Andersson, N.; Hamel, C.; Porter, A.C.; Tugwell, P.; Moher, D.; Bouter, L.M. Development of AMSTAR: A Measurement Tool to Assess the Methodological Quality of Systematic Reviews. BMC medical research methodology 2007, 7, 1-7.

[19] Driller, M.; Mackay, K.; Mills, B.; Tavares, F. Tissue Flossing on Ankle Range of Motion, Jump and Sprint Performance: A Follow-up Study. Physical Therapy in Sport 2017, 28, 29-33.

[20] Kiefer, B.N.; Lemarr, K.E.; Enriquez, C.C.; Tivener, K.A.; Daniel, T. A Pilot Study: Perceptual Effects of the Voodoo Floss Band on Glenohumeral Flexibility. International Journal of Athletic Therapy and Training 2017, 22, 29-33.
[21] Ross, S.; Kandassamy, G. The Effects of 'Tack and Floss' Active Joint Mobilisation on Ankle Dorsiflexion Range of Motion Using Voodoo Floss Bands. Journal of Physical Therapy 2017, 1-22.

[22] Stevenson, P.J.; Stevenson, R.K.; Duarte, K.W. Acute Effects of the Voodoo Flossing Band on Ankle Range of Motion. Journal of Medical Biomedical and Applied Sciences 2019, 7, 244-253.

[23] Williams, Z.; Carlson, S.; Rife, G. Comparing the Effects of Tissue Flossing and Instrument Assisted Soft Tissue Mobilization on Ankle Dorsiflexion. In Proceedings of the Proceedings of the The Research and Scholarship Symposium; Cedarville, OH, 2019; Vol. 3.

[24] Galis, J.; Cooper, D.J. Application of a Floss Band at Differing Pressure Levels: Effects at the Ankle Joint. Journal of Strength and Conditioning Research 2020, Epub ahead of print.

[25] Kaneda, H.; Takahira, N.; Tsuda, K.; Tozaki, K.; Kudo, S.; Takahashi, Y.; Sasaki, S.; Kenmoku, T. Effects of Tissue Flossing and Dynamic Stretching on Hamstring Muscles Function. Journal of sports science \& medicine 2020, 19, 681-689.

[26] Pisz, A.; Kralova, K.; Blazek, D.; Golas, A.; Stastny, P. Meta-Analyses of the Effect of Flossing on Ankle Range of Motion and Power Jump Performance. Baltic Journal of Health and Physical Activity 2020, 12, 19-26.

[27] Vogrin, M.; Novak, F.; Licen, T.; Greiner, N.; Mikl, S.; Kalc, M. Acute Effects of Tissue Flossing on Ankle Range of Motion and Tensiomyography Parameters. Journal of sport rehabilitation 2020, 30, 129-135.

[28] Hodeaux, K. The Effect of Floss Bands on Elbow Range of Motion in Tennis Players. Master Thesis, University of Arkansas: Fayetteville, AR, 2017.

[29] McCallum, A. The Effect of Chiropractic Manipulation and Ankle Flossing on Ankle Range Or Motion in Patients with Chronic Ankle Inversion Injury. PhD Thesis, University of Johannesburg: Johannesburg, 2020.

[30] Stastny, P. Meta-Analyses of the Effect of Flossing on Ankle Range of Motion and Power Jump Performance. Baltic Journal of Health and Physical Activity 2020, 12, 19-26.

[31] Mills, G.E. Action Research: A Guide for the Teacher Researcher; Prentice-Hall, Inc: Upper Saddle River, NJ, 2000;

[32] Konrad, A.; Bernsteiner, D.; Budini, F.; Reiner, M.M.; Glashüttner, C.; Berger, C.; Tilp, M. Tissue Flossing of the Thigh Increases Isometric Strength Acutely but Has No Effects on Flexibility or Jump Height. European Journal of Sport Science 2020, 1-11.

[33] Klingler, W.; Velders, M.; Hoppe, K.; Pedro, M.; Schleip, R. Clinical Relevance of Fascial Tissue and Dysfunctions. Current pain and headache reports 2014, 18, 1-7. 